\title{
The Non-Peptidic HIV Protease Inhibitor Tipranavir and Two Synthetic Peptidomimetics (TS98 and TS102) Modulate Pneumocystis carinii Growth and Proteasome Activity of HEL299 Cell Line
}

\author{
FRANCESCA MAZZA, ${ }^{\mathrm{a}}$ ELISA TRONCONI, ${ }^{\mathrm{a}}$ ANTONELLA VALERIO, ${ }^{\mathrm{a}}$ MARCUS GROETTRUP, ${ }^{\mathrm{b}}$ MARCEL KREMER, \\ ALESSANDRO TOSSI, ${ }^{\mathrm{c}}$ FABIO BENEDETTI ${ }^{\mathrm{c}}{ }_{\text {ANTONIETTA CARGNEL }}^{\mathrm{a}}$ and CHIARA ATZORI ${ }^{\mathrm{a}}$ \\ ${ }^{\mathrm{a} I I}$ Department of Infectious Diseases, L. Sacco Hospital, Milan, Italy, and \\ ${ }^{\mathrm{b}}$ Division of Immunology, Department of Biology, University of Konstanz, Konstanz, Germany, and
}

${ }^{\mathrm{c}}$ University of Trieste, Trieste, Italy.

$\mathrm{T}$

HE clinical efficacy of highly active antiretroviral therapy

(HAART) containing HIV protease inhibitors against opportunistic infections is now well known (Sepkowitz 1998). Widely used HIV protease inhibitors (including Saquinavir, Ritonavir, Indinavir, Nelfinavir, Amprenavir, Lopinavir, and Atazanavir) are compounds with structures that mimic the local topography around an amide bond. They are low MW peptidomimetics (MW $<1,000 \mathrm{Da}$ ) containing transition-state isostere (hydroxyethylamine and hydroxyethylene) that match the peptide backbone atom-for-atom and form important contacts with the HIV protease binding-site (Ripka and Rich 1998). We previously demonstrated that the majority of available HIV protease inhibitors partially and non-specifically reduced rat-derived Pneumocystis carinii growth in vitro when tested at clinically administered concentrations (Atzori et al. 2000, 2001; Mazza 2003).

In this study we evaluated the anti-Pneumocystis activity of three other compounds, Tipranavir, TS98, and TS102. TS98 and TS102 are small peptidomimetics that are structurally and functionally correlated with clinical HIV protease inhibitors. TS98 and TS102 were designed by taking into account the binding site of HIV-1 and Candida albicans (Sap2) proteases (Tossi et al. 2003). Tipranavir is the first compound of a new class of non-peptidic HIV protease inhibitors based on novel templates which, though appearing unrelated to the original peptides, contain the necessary groups positioned on a non-peptidic scaffold that serves as a topographical mimetic. This kind of molecules represents an ideal peptidomimetic in that their non-peptidic nature makes them recalcitrant to proteolytic degradation and hence have increased bioavailability (Ripka and Rich 1998). Because of the lack of a continuous in vitro culture system for $P$. carinii, we assayed Tipranavir, TS98, and TS102 in a monoxenic culture system containing $P$. carinii isolated from infected rat lungs and human embryonic lung cells (HEL299) feeder cells. To understand if the effects observed on $P$. carinii proliferation in vitro were related to modulation of feeder cell proteasomes, we performed examined the effect of the three compounds on intact HEL299 cells and the 20S proteasome isolated from them (Groettrup et al. 1995).

The $26 \mathrm{~S}$ proteasome is a multifunctional proteolytic complex of approximately $2,000 \mathrm{kDa}$ consisting of two subcomplexes. These include the 20S proteasome and the 19S particle that serve catalytic and regulatory functions, respectively. The $26 \mathrm{~S}$ proteasome is able to degrade proteins with almost complete specificity for ubiquitin-conjugated proteins, and its catalytic activity depends on ATP. In vivo, the $20 \mathrm{~S}$ proteasome is likely to exist as a single particle or in combination with the $19 \mathrm{~S}$ regulator. It can also combine with another particle, $11 \mathrm{~S}$. $11 \mathrm{~S}$ plays a regulatory

Corresponding Author: C. Atzori, II Department of Infectious Diseases, L. Sacco Hospital, via G.B. Grassi 74, 20157 Milan, Italy-Telephone number: +1-39-02-39042953; FAX number: +1-39-0238200909; e-mail: c.atzori@hsacco.it role in producing the hybrid $19 \mathrm{~S}-20 \mathrm{~S}-11 \mathrm{~S}$ particle, which requires ATP for its assembly and retains the ability to degrade ubiquitinated conjugates. Proteasomes purified from different tissues and species are approximately $700 \mathrm{kDa}$ in size and are comprised of four staked rings, each containing multiple subunits. This particle designated as the $20 \mathrm{~S}$ proteasome retains peptidase activity without requiring ATP and is unable by itself to degrade proteins combined with ubiquitin molecules (Piccinini, Mostert, and Rinaudo 2003).

\section{MATERIALS AND METHODS}

Pneumocystis carinii culture. Trophic forms of $P$. carinii were obtained from infected lungs of immunosuppressed transtracheally infected rats and added to monolayer cultlures of HEL299 (ATCC CCL137). Cultures were untreated (negative control) or treated with Tipranavir $(0.35,0.7,3.5,7.0 \mu \mathrm{M})$, TS98 $(0.18,1.8,9.0 \mu \mathrm{M})$, or TS102 $(0.16,1.6,7.8 \mu \mathrm{M})$. Co-trimoxazole $(250 \mu \mathrm{g} / \mathrm{ml})$ was used as positive control. At $1,3,5$, and 7 days after inoculation, the cultures were agitated and a $10 \mu \mathrm{l}$ sample of the culture supernatant was removed, air dried within a $1 \mathrm{~cm}^{2}$ area etched on a glass slide, and stained with Diff-Quik. Organisms were enumerated using a $100 \times$ objective. Values were expressed as the number of $P$. carinii per microscopic field and were analyzed by a two-way ANOVA test and the Bonferroni correction as a post hoc test.

Fluorometric assays of intact HEL299 cells and the $20 S$ proteasome purified from them. HEL299 20S proteasome was purified by established protocols (Groettrup et al. 1995). The protein complex was incubated with the fluorogenic substrate Suc-LLVY-AMC, specific for chymotrypsin-like activity, and with Tipranavir, TS98, or TS102 at the same concentrations used on the $P$. carinii proliferation experiments described above. Assays were performed with or without Lactacystin $(10 \mu \mathrm{M})$, a proteasome-specific inhibitor. Proteasome activity was measured after $60-90 \mathrm{~min}$.

The same assay was used to analyze intact HEL299 monolayers that had been exposed to the drugs; proteasome activity in these cells was measured on days 1,4 , and 8 . MeO-Suc-GLF-AMC, a membrane-permeant fluorogenic substrate specific for chymotrypsin-like activity was used in these assays. Lactacystin $(30 \mu \mathrm{M})$ was used as positive control. Results were analyzed using a two-way ANOVA test and the Bonferroni correction as a post hoc test.

\section{RESULTS AND DISCUSSION}

Tipranavir at $0.7,3.5$, and $7.0 \mu \mathrm{M}$ significantly reduced P. carinii proliferation in vitro (Fig. 1). Both TS98 and TS102 also had significant inhibitory effects on $P$. carinii at all concentrations tested. The effect these three compounds on $P$. carinii proliferation in vitro agrees with the results previously obtained 


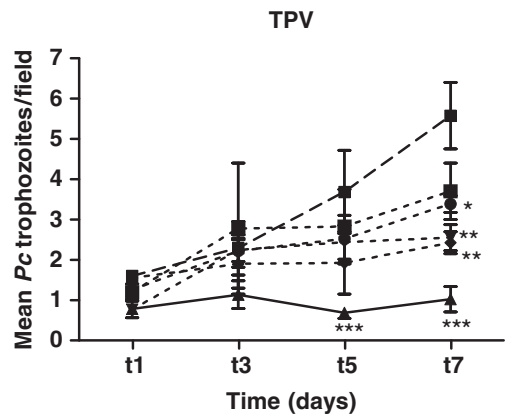

$\%$ growth inhibition vs Pc untreated at $t 7$

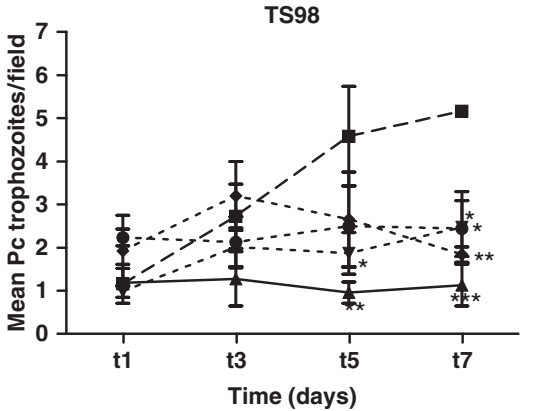

$\%$ growth inhibition vs $\mathrm{Pc}$ untreated at $\mathrm{t} 7$

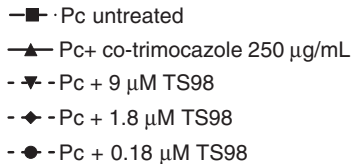

$$
\begin{gathered}
\text { / } \\
78 \% \\
52 \% \\
64 \% \\
53 \%
\end{gathered}
$$

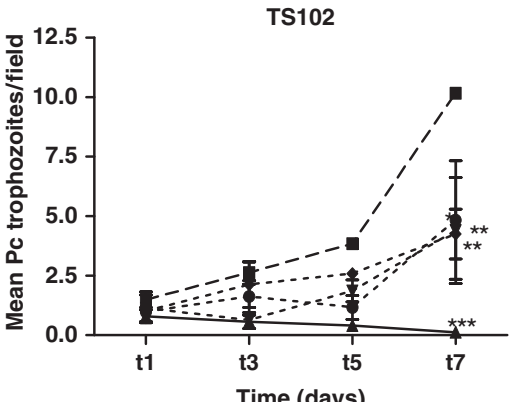

$\%$ growth inhibition vs Pc untreated at $t 7$

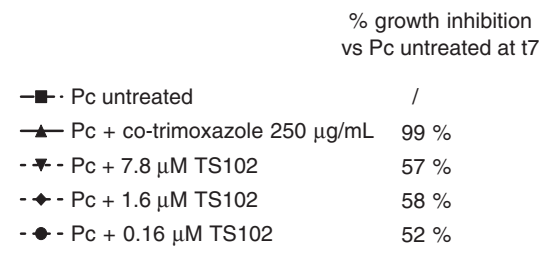

Fig. 1. Effect of Tipranavir, TS98, and TS102 on proliferation of Pneumocystis carinii (Pc) trophic forms cultured on human embryonic lung cells (HEL299) confluent monolayers. The organisms in culture supernates were counted at the times shown. ${ }^{*} P<0.05,{ }^{* *} P<0.01,{ }^{* * *} P<0.001$.
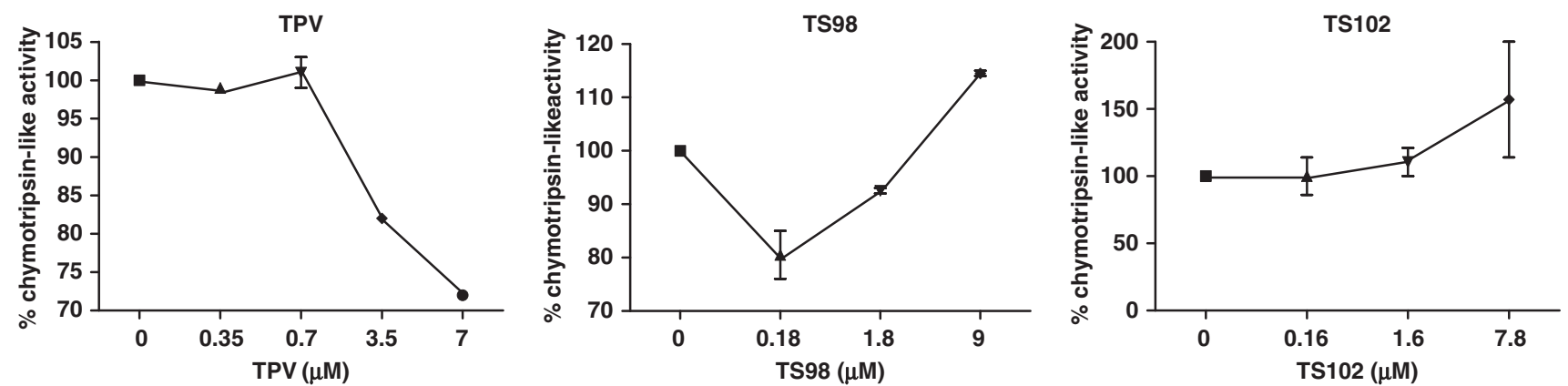

Fig. 2. Chymotrypsin-like activity of $20 \mathrm{~S}$ proteasome purified from human embryonic lung cells (HEL299 cells) and treated with Tipranavir, TS98, or TS102. Values are expressed as percentage of enzyme activity in untreated control (100\%).
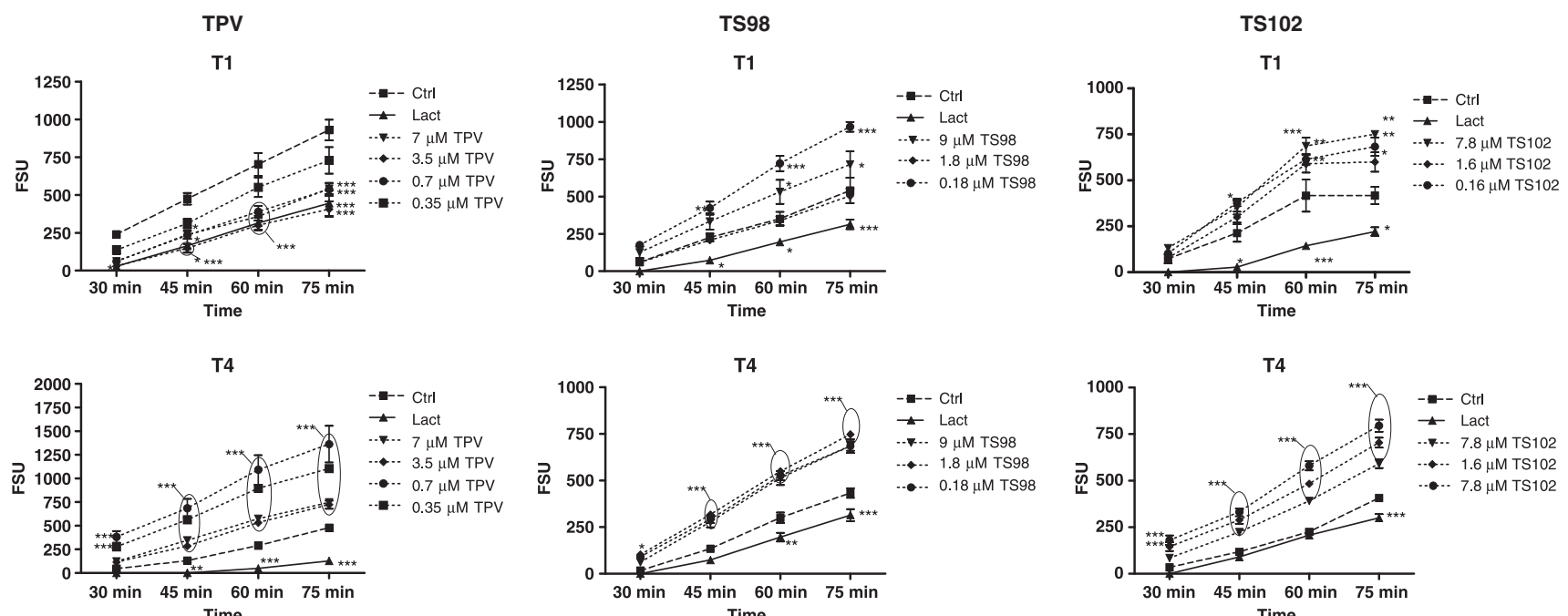

Fig. 3. Effects of Tipranavir, TS98, and TS102 on the chymotrypsin-like activity of the 20S proteasome in intact human embryonic lung cells (HEL299 cells). Data represent the values of fluorescence measured at various times during the assay. Enzyme activity analyses performed on day 1 and day 4 of incubation are shown. ${ }^{*} P<0.05,{ }^{* *} P<0.01,{ }^{* * *} P<0.001$. 
when many HIV protease inhibitors were tested at clinically prescribed concentrations. These results suggest that structurally different molecules (peptidic and non-peptidic) can give rise to the same final outcome on inhibiting $P$. carinii growth.

The chymotrypsin-like activity of the $20 \mathrm{~S}$ proteasome purified from the HEL299 cells was enhanced by TS98 and TS102 at the higher concentrations tested (Fig. 2). In contrast, this activity was reduced using 3.5 and $7.0 \mu \mathrm{M}$ Tipranavir. This phenomenon could be explained by a "two-site modifier" kinetic model (Schmidtke et al. 2000): in which Tipranavir down-regulates the proteasome activity by interaction with a chymotrypsin-specific active site, whereas TS98 and TS102 up-regulates a proteasome modulation site distinct from the catalytic site.

The fluorometric assay performed on intact HEL299 cells indicated that TS98 and TS102 enhanced proteasome activity in these cells (Fig. 3), which verifies the observations made on isolated proteasome preparations. However, after 1 day (Fig. 3, T1) of incubation with Tipranavir we observed an inhibitory effect of the chymotrypsin-like activity. According to the "two-modifier" kinetic model, the mechanism for this reduction could be competitive inhibition between the drug and the fluorogenic peptide for binding to the enzyme active site. After this initial reduction, there was an increase in proteasome enzyme activity (Fig. 3, T4). At T8 (8 days after incubation with the compound) we did not observed significant differences between the samples incubated with Tipranavir and the untreated control. The response of HEL299 cell after 4 days of exposure to Tipranavir could then be explained by increased enzyme activity resulting from over-expression of many genes coding for the proteasome. Hepatocytes of rats treated with Ritonavir have been reported to exhibit a similar response to this drug (Waring et al. 2005). The effect of protease inhibitors on modulating the HEL299 proteasome and the observed inhibition of $P$. carinii proliferation when these organisms are treated with drugs and co-cultured with feeder cells requires further study to fully understand the interactions between the two cell types in the same culture system.

\section{LITERATURE CITED}

Atzori, C., Angeli, E., Mainini, A., Agostoni, F., Micheli, V. \& Cargnel, A. 2000. In vitro activity of human immunodeficiency virus protease inihibitors against Pneumocystis carinii. J. Infect. Dis., 181:1629-1634.

Atzori, C., Fantoni, G., Valerio, A. \& Cargnel, A. 2001. In vitro activity of amprenavir against Pneumocystis carinii. Int. J. Antimicrob. Agents, 18:271-273.

Groettrup, M., Ruppert, T., Kuehn, L., Seeger, M., Standera, S., Koszinowski, U. \& Kloetzel, P. M. 1995. The interferon- $\gamma$-inducible $11 \mathrm{~S}$ regulator (PA28) and the LMP2/LMP7 subunits govern the peptide production by the $20 \mathrm{~S}$ proteasome in vitro. J. Biol. Chem., 270:2380823815.

Mazza, F. 2002-2003. Study of the effect of the HIV protease inhibitors lopinavir and atazanavir on in vitro Pneumocystis Carinii growth. Dissertation, University of Milano-Bicocca, Milan, Italy, 78 p.

Piccinini, M., Mostert, M. \& Rinaudo, M. T. 2003. Proteasomes as drug targets. Cur. Drug Targets, 4:657-671.

Ripka, A. S. \& Rich, D. H. 1998. Peptidomimetic design. Cur. Opin. Chem. Biol., 2:441-452.

Sepkowitz, K. A. 1998. Effect of HAART on natural history of AIDSrelated opportunistic disorders. Lancet, 351:228-230.

Schmidtke, G., Emch, S., Groettrup, M. \& Holzhutter, H. G. 2000. Evidence for the existence of a non-catalytic modifier site of peptide hydrolysis by the 20S proteasome. J. Biol. Chem., 275:22056-22063.

Tossi, A., Benedetti, F., Norbedo, S., Skrbec, D., Berti, F. \& Romeo, D. 2003. Small hydroxyethylene-based peptidomimetics inhibiting both HIV-1 and C. albicans aspartic proteases. Bioorg. Med. Chem., 11:4719-4727.

Waring, J., Ciurlionis, R., Marsh, K., Klein, L., Degoey, D., Randolph, J., Spear, B. \& Kempf, D. 2005. Identification of proteasome gene regulation in rat model for HIV-induced hyperlipidemia using microarray analysis. Abstract 839. Conference on Retroviruses and Opportunistic Infections. 